\title{
RATIONAL CONNECTIVITY AND SECTIONS OF FAMILIES OVER CURVES
}

\author{
TOM GRABER, JOE HARRIS, BARRY MAZUR, AND JASON STARR
}

\section{INTRODUCTION}

1.1. Statement of results. In GHS, it is proved that a one-parameter family of rationally connected varieties always has a rational section: explicitly, we have the

Theorem 1.1. Let $\pi: X \rightarrow B$ be a proper morphism of complex varieties, with $B$ a smooth curve. If the general fiber of $\pi$ is rationally connected, then $\pi$ has a section (recall a complex variety $V$ is called rationally connected if any two points on $V$ lie on the image of some map $h: \mathbb{P}^{1} \rightarrow V$ ).

The goal of this paper is to state and prove a converse to this statement (c.f. Theorem 1.3 below).

We should first of all discuss what we mean by this, inasmuch as the literal converse of Theorem 1.1 is clearly false. To this end, let's focus on the question: under what circumstances does a family $\pi: X \rightarrow B$ of varieties have the property that its restriction to a general curve $C \subset B$ has a section?

This is certainly the case if the family $\pi: X \rightarrow B$ has a global rational section. It is also the case by Theorem 1.1 if the general fiber of $\pi$ is rationally connected, and by extension it's the case if $X$ contains a subvariety $Z \subset X$ dominating $B$ and whose fiber over a general point of $B$ is rationally connected. (We can think of the case where the family $\pi: X \rightarrow B$ has a global rational section as a special case of this, a single point being a rationally connected variety!) In this paper, we will prove that in fact these are the only circumstances under which it may occur. To make this claim precise, we start by making the definition

Definition 1.2. Let $\pi: X \rightarrow B$ be an arbitrary morphism of complex varieties. By a pseudosection of $\pi$ we will mean a subvariety $Z \subset X$ such that the restriction $\left.\pi\right|_{Z}: Z \rightarrow B$ is dominant with rationally connected general fiber.

Our main result is

Theorem 1.3. Let $B$ be any variety. For every positive integer $d$ there exists a bounded family $\mathcal{H}_{d}$ of maps $h: C \rightarrow B$ from smooth irreducible curves to $B$ such that for any proper morphism $\pi: X \rightarrow B$ of relative dimension $d$ or less, if $h: C \rightarrow B$ is a map parametrized by a very general point of $\mathcal{H}_{d}$, the pullback

$$
\pi_{C}: X_{C}=X \times{ }_{B} C \rightarrow C
$$

has a section if and only if $\pi$ has a pseudosection.

Date: August 24, 2021.

T.G. was partially supported by an NSF postdoctoral fellowship.

J.S. was partially supported by NSF grant DMS-0201423. 
If $B$ is normal and quasi-projective, we can take $\mathcal{H}_{d}$ to be the family of smooth linear sections of $B$ under a sufficiently positive projective embedding.

What we are saying here, in other words, is that the class of rationally connected varieties is the largest class for which the statement of Theorem 1.1 holds: if we have any other family of varieties $\pi: X \rightarrow B$ satisfying the condition that every one-parameter subfamily has a section, it does so by virtue of the fact that $X$ contains a family of rationally connected varieties.

As a corollary of this, we will at the end of the paper settle a question left hanging in [GHS]: whether or not the statement of Theorem 1.1 holds for the larger class of $\mathcal{O}$-acyclic varieties - that is, varieties $X$ with $H^{i}(X, \mathcal{O})=0$ for all $i>0$. In fact, it was suggested by Serre in a letter to Grothendieck ([GS], p. 152) that this might be the case (though Serre immediately adds that it is "sans doute trop optimiste"). In Section 5, we show this does not hold: specifically, by applying Theorem 1.3 to the universal family over a parameter space of Enriques surfaces with a particular polarization, we will deduce the

Corollary 1.4. There exists a one-parameter family $X \rightarrow B$ of Enriques surfaces with no rational section.

\section{Stable maps and stable Sections}

Our proof of Theorem 1.3 involves an induction on the relative dimension of $f: X \rightarrow B$ where the base case (i.e. fiber dimension zero) is proved by a version of the Lefschetz hyperplane theorem. In the course of the proof we will need to use specializations of irreducible curves in $B$. There are several possible compactifications of the Chow variety of irreducible curves in $B$, but the one we will use is the Kontsevich space of stable maps. The reader who is unfamiliar with stable maps is referred to the article [FP]. But the reader will not be far off if he thinks of a stable map to $X$ as a map $h: C \rightarrow X$ for which $C$ is a connected, projective curve which has at-worst-nodes as singularities i.e. every point of $C$ is either smooth or is formally isomorphic to the origin on the curve $\left\{(x, y) \in \mathbb{A}^{2}: x y=0\right\}$. There is an equivalence relation on stable maps which is the obvious one, and the Kontsevich moduli space of stable maps is the corresponding moduli space of equivalence classes of stable maps.

We let $\bar{M}_{g}(X, \beta)$ denote the Kontsevich space of stable maps $h: C \rightarrow X$ such that $C$ has arithmetic genus $g$ and such that the push-forward fundamental class $h_{*}[C]$ equals $\beta \in H_{2}(X, \mathbb{Z})$. This is a proper Deligne-Mumford stack with projective coarse moduli scheme (c.f. [FP]). We will often not need the decorations, so let $\bar{M}(X):=\cup_{g, \beta} \bar{M}_{g}(X, \beta)$ denote the space of all stable maps to $X$.

Definition 2.1. If $h: C \rightarrow X$ and $h^{\prime}: C^{\prime} \rightarrow X$ are stable maps, we say that $h^{\prime}$ is a submap of $h$ if there is a factorization $h^{\prime}=h \circ i$ for some $i: C^{\prime} \rightarrow C$.

Note in the above definition we do not assume that $i$ is an embedding. The following definition makes precise what it means for one stable map to be a submap of a specialization of another stable map.

Definition 2.2. Given two irreducible families of stable maps, $D$ and $E$, we say that $D$ dominates $E$ if every map parametrized by $E$ can be realized as a submap 
of a specialization of maps parametrized by $D$. Precisely, for any point $e \in E$ parametrizing a stable map $h: C \rightarrow X$, we can find a family of stable maps over $\operatorname{Spec}(\mathbb{C}[[t]])$ such that $h$ is a submap of the map over the special fiber, and the map over the generic fiber is pulled back by a morphism from $\operatorname{Spec}(\mathbb{C}((t)))$ to $D$. If $D$ and $E$ are reducible, we say $D$ dominates $E$ if every component of $D$ dominates every component of $E$.

We need a criterion for when a family of stable maps to a projective variety is dominated by a family of embedded complete intersection curves. We will repeatedly make use of the following criterion.

Lemma 2.3. Suppose $E$ is a family of stable maps to a normal, projective variety $B$ such that for the general map $h: C \rightarrow B$ parametrized by $E$, there is an open subset $U \subset B$ contained in the smooth locus of $B$ and such that:

(1) $h^{-1}(U)$ is dense in $C$,

(2) $h^{-1}(U)$ contains all the nodes of $C$, and

(3) the restricted map $h^{-1}(U) \rightarrow U$ is a closed embedding.

Then $E$ is dominated by the family of one dimensional linear sections of $B$ under a sufficiently positive projective embedding.

Proof.

Let $h: C \rightarrow B$ be a general element of our family. We embed $B$ in a projective space and find an integer $a$ such that the ideal of the reduced image curve, $h(C)$ is generated by polynomials of degree $a$. The sufficiently positive embedding required is the ath Veronese reembedding. Now we know that we can realize $h(C)$ as an intersection of hyperplanes. If we choose $\operatorname{dim}(B)-1$ generic hyperplane sections of $B$ which contain $h(C)$, their intersection will be a generically reduced curve $C^{\prime}$ which contains $h(C)$ as a subcurve. Choosing the hyperplanes so that they generate the ideal of $h(C)$ at each of the images of the nodes of $C$, and after possibly shrinking $U$ (so that it still satisfies our hypotheses), we can arrange that $h^{-1}(U) \rightarrow U \cap C^{\prime}$ is an isomorphism.

Now we choose any one parameter family of smooth complete intersections in $B$ whose flat limit is $C^{\prime}$, i.e. a morphism from $\operatorname{Spec}(\mathbb{C}[[t]])$ to the Hilbert scheme of complete intersections in $B$ whose general fiber maps to a smooth complete intersection and whose special fiber maps to $C^{\prime}$. We think of the general fiber as a stable map and perform stable reduction to the corresponding map $\operatorname{Spec}(\mathbb{C}[[t]]) \rightarrow$ $\bar{M}(B)$. Denote the special fiber of the stable reduction by $\bar{h}: \bar{C} \rightarrow B$. Since $U \cap C^{\prime}$ is already at-worst-nodal and stable (since it is an embedding), there is an open subset $V \subset \bar{C}$ such that $\bar{h}: V \rightarrow U \cap C^{\prime}$ is an isomorphism. In other words, we have a factorization $i: h^{-1}(U) \rightarrow \bar{C}$ of $h: h^{-1}(U) \rightarrow B$. Since every point of $C$ in $C-h^{-1}(U)$ is smooth, we can apply the valuative criterion of properness to extend this factorization to a morphism $i: C \rightarrow \bar{C}$. So $h: C \rightarrow B$ is a submap of $\bar{h}: \bar{C} \rightarrow B$, which shows that $E$ is dominated by the family of smooth curves in $B$ which are complete intersections of $d-1$ hyperplanes.

We remark that the lemma above is not the most general result, but to prove a stronger version would lead us too far astray. We leave it to the interested reader 
to prove that in the above lemma it suffices to assume that for the general map $h: C \rightarrow B$ parametrized by $E$, the preimage of the smooth locus, $h^{-1}\left(B_{\mathrm{smooth}}\right)$, is a dense open set which contains every node of $C$.

Although our main concern is to understand sections of a map $\pi: X \rightarrow B$ over smooth curves in $B$, the specialization methods we use force us to consider the more general notion of stable maps. Similarly, we need to replace the notion of section over a curve with an object which specializes as we specialize the base curve. To explain this notion, we first recall a standard construction.

Given a morphism of projective schemes $\pi: X \rightarrow B$, there is an induced map on Kontsevich spaces, $\bar{M}(\pi): \bar{M}(X) \rightarrow \bar{M}(B)$ (technically we must restrict to those stable maps with $g>1$ or with $\beta \neq 0$, but this will always be the case for us). This map takes the moduli point $f: C \rightarrow X$ to the point $\pi \circ f: \tilde{C} \rightarrow B$, where $\tilde{C}$ is the stabilization of $C$ relative to the morphism $\pi \circ f$. It is obtained by contracting those components of $C$ of genus 0 which are contracted by $\pi \circ f$ and meet the rest of $C$ in fewer than 3 points.

Definition 2.4. Given a morphism $\pi: X \rightarrow B$, and a stable map $h: C \rightarrow B$, we define a stable section of $\pi$ over $[h]$ to be a stable map $\tilde{h}: \tilde{C} \rightarrow X$ such that $\bar{M}(\pi)([\tilde{h}])=[h]$.

Notice that for a stable section $\tilde{h}$, the class $\pi_{*}(\tilde{h})_{*}[\tilde{C}]$ is just $h_{*}[C]$ and $g\left(C^{\prime}\right)=$ $g(C)$. Since $h$ is a stable map, either $g(C)>1$ or $h_{*}[C]$ is nonzero, i.e. the map $\bar{M}(\pi)$ really is defined in a neighborhood of $[\tilde{h}]$.

If $C$ is a smooth curve in $B$, then a stable section of $\pi$ over $C$ is simply a section of $\pi$ over $C$ with some trees of $\pi$-contracted rational curves in $X$ attached. The notion is more interesting when $C$ has nodes. In this case, the existence of a stable section over $C$ does not guarantee the existence of any sections over $C$ whatsoever. For example, a typical stable section $\tilde{h}$ over a curve $C=C_{1} \cup C_{2}$, where $t_{1} \in C_{1}$ is glued to $t_{2} \in C_{2}$, would consist of sections $\tilde{h}_{1}$ and $\tilde{h_{2}}$ of $\pi$ over $C_{1}$ and $C_{2}$ separately together with a tree of $\pi$-contracted rational curves in $X$ joining $\tilde{h}_{1}\left(t_{1}\right)$ to $\tilde{h}_{2}\left(t_{2}\right)$ (and some trees of $\pi$-contracted rational curves attached elsewhere). Such rational curves are exactly the sort which are contracted under the stabilization process associated with $\bar{M}(\pi)$.

The point of this definition is that given a family of curves in the base $B$ specializing to some stable map $h: C \rightarrow B$ (possibly reducible), and given an honest section over the generic curve in this family, then we cannot conclude the existence of a section of $\pi$ over $h$, but we do conclude the existence of a stable section of $\pi$ over $h$. In other words, the existence of a stable section is preserved under specialization. This follows immediately from the properness of the irreducible components of $\bar{M}(X)$. Another elementary fact is that if $h^{\prime}$ is a submap of $h$, then the existence of a stable section over $h$ implies the existence of a stable section over $h^{\prime}$. Taken together, we get the following 
Lemma 2.5. If $D$ and $E$ are families of stable maps to $B$, with $D$ dominating $E$ and if a general map parametrized by $D$ admits a stable section, then so does every map parametrized by $E$.

As an application, we can strengthen the easy direction of our main theorem.

Proposition 2.6. If $\pi: X \rightarrow B$ is a morphism of projective varieties with $B$ smooth and if $\pi$ admits a pseudosection, then for any smooth curve $C$, and for any morphism $h: C \rightarrow B$, the pullback family $X_{C} \rightarrow C$ admits a section.

Proof.

Since any map from a smooth curve factors through the normalization of its image, it suffices to prove this statement for maps birational onto their image. Let $Z$ be a pseudosection of $\pi$. We already know by Theorem 1.1 that the proposition is true for any smooth curve such that the general fiber of $Z$ over the curve is rationally connected. In particular it holds for a generic complete intersection curve in $B$. By Lemma 2.3, our map $h$ can be realized as a submap of a limit of such curves. Then Lemma 2.5 implies that $\pi$ admits a stable section over $[h]$. Since $C$ is smooth, this implies that $X_{C}$ admits a section over $C$.

\section{Proof of MAIN THEOREM}

In our proof of Theorem 1.3, we begin by assuming that $B$ is normal and that both $X$ and $B$ are projective. After handling this "special" case, we give the (easy) argument which reduces the general case to the special case.

We will prove the theorem by induction on the relative dimension of $X$ over $B$. We start with the case of relative dimension zero.

Proposition 3.1. Let $B \subset \mathbb{P}^{n}$ be a normal variety and $\pi: X \rightarrow B$ a generically finite proper morphism. Then $\pi$ admits a rational section if and only if $\pi$ admits a section when restricted to a general one dimensional linear section of $B$.

Proof.

Let $B_{0} \subset B$ be an open subset such that the restriction of $\pi$ to $X_{0}=\pi^{-1}\left(B_{0}\right)$ is finite and étale of some degree $k$. Choose a one dimensional linear section of $B$ which is a smooth curve $C$, such that the natural map $\pi_{1}\left(C \cap B_{0}\right) \rightarrow \pi_{1}\left(B_{0}\right)$ is surjective. Such a $C$ exists by $\overrightarrow{\mathrm{GM}}$. In fact, any smooth linear section which meets $B \backslash B_{0}$ suitably transversally will do. Define $C_{0}=C \cap B_{0}$, and choose any point $c_{0} \in C_{0}$. Choosing an ordering of the points in $\pi^{-1}\left(c_{0}\right)$ gives us a natural monodromy representation $\rho: \pi_{1}\left(B_{0}, c_{0}\right) \rightarrow S_{k}$ where $S_{k}$ is the symmetric group on $k$ letters. The monodromy representation associated to the étale cover $\pi^{-1}\left(C_{0}\right) \rightarrow C_{0}$ is just the composition $\rho \circ i_{*}$ where $i: C_{0} \rightarrow B_{0}$ is the inclusion. The statement that $\pi$ admits a section over $C$ is equivalent to this étale cover of $C_{0}$ admitting a section, which is equivalent to asking for the image of $\rho \circ i_{*}$ to be contained in the stabilizer of an element. Since $i_{*}$ is surjective, this is equivalent to asking for the image of $\rho$ to be contained in the stabilizer of an element. This in turn is equivalent to the existence of a section of $X_{0}$ over $B_{0}$, i.e. a rational section of $\pi$. 
In handling the case of positive relative dimension, one of the main ingredients needed is the following bend-and-break lemma for sections.

Lemma 3.2. Let $\pi: X \rightarrow C$ be a morphism with $C$ a smooth curve. Let $p \in X$ be an arbitrary point. If there is a positive dimensional family of sections of $\pi$ passing through $p$, then there is a rational curve in $X$ passing through $p$ which is contracted by $\pi$.

Proof.

Let $q=\pi(p)$. Suppose we have a one parameter family of sections passing through $p$. This gives us a rational map $f: B \times C \rightarrow X$ over $C$ whose restriction to a general fiber $\{b\} \times C$ is a section passing through $p$. Suppose, by way of contradiction, that $f$ is a regular morphism in a neighborhood of $B \times\{q\}$. Since $f$ contracts $B \times\{q\}$, by the rigidity lemma it also contracts $B \times\{c\}$ for all $c \in C$, i.e. our family is constant which contradicts that it is positive dimensional. Hence, $f$ is not regular near $B \times\{q\}$. So there is at least one point of indeterminacy in $B \times\{q\}$. We may form the minimal blow-up of $B \times C$ necessary to resolve the indeterminacy locus of $f$. The exceptional divisor of this blow-up is a tree of rational curves which intersects the proper transform of $B \times\{q\}$ and which is mapped to a tree of $\pi$ contracted rational curves in $X$. Therefore some rational curve in the exceptional divisor maps to a $\pi$-contracted rational curve which meets $p$.

We will apply this lemma in two ways. The first application is to get a uniform bound on the dimensions of spaces of sections. First we need a definition.

Definition 3.3. If $\pi: X \rightarrow B$ is a morphism, we define the rational curve locus, $V(\pi)$, to be the union of all $\pi$-contracted rational curves in $X$.

Let us pause to describe what sort of object $V(\pi)$ is. Fixing an ample divisor $H$ on $X$, then for each integer $d$ there is a finite type Chow variety (or Hilbert scheme, or Kontsevich space of stable maps) parametrizing $\pi$-contracted rational curves whose $H$-degree is $d$. Over each such Chow variety, there is a universal family of $\pi$-contracted rational curves along with a map to $X$. And $V(\pi)$ is simply the union over all $d$ of the image of this map from the universal family to $X$. We think of $V(\pi)$ as just a set, but we constantly use the fact that $V(\pi)$ is a set which is a countable union of subvarieties of $X$.

Here is our uniform bound on the dimensions of spaces of sections.

Lemma 3.4. If $\pi: X \rightarrow C$ is a morphism of relative dimension $d$ and if $\Sigma$ is a family of sections of $\pi$ such that a general section parametrized by $\Sigma$ is not contained in $V(\pi)$, then $\operatorname{dim}(\Sigma) \leq d$.

Proof.

Choose a very general point $c$ of $C$. Let $e v_{c}: \Sigma \rightarrow X_{c}$ be the map which evaluates a section at $c$. Our hypotheses ensure that $\operatorname{dim}\left(X_{c}\right) \leq d$ and that $e v_{c}(\Sigma) \not \subset V(\pi)$. Lemma 3.2 then implies that $e v_{c}$ is generically finite onto its image, yielding the desired bound. 
Definition 3.5. Let $L$ denote the family of smooth linear sections of $B$ under some projective embedding. We define the family of triangles, $T(L) \subset \bar{M}(B)$ to be the family of all stable maps which look like a triangle with sides in $L$. That is, we consider the family of morphisms from a curve of the form $C=C_{1} \cup C_{2} \cup C_{3}$ such that every pair of components of the domain curve meet in a single point (and these intersection points are pairwise distinct), and such that each $\left.f\right|_{C_{i}} i=1,2,3$ is an embedding onto a curve parametrized by $L$.

The parameter space $T(L)$ is irreducible, and also the total space of the universal family of curves over $T(L)$ is irreducible. Note also that a general stable map parametrized by $T(L)$ satisfies the hypotheses of Lemma 2.3.

Our second application of Lemma 3.2 is in the proof of the following lemma, which is the main step in the proof of Theorem 1.3.

Lemma 3.6. Let $\pi: X \rightarrow B$ be a projective morphism to a normal variety, and let $p \in X$ be any point such that $p \notin V(\pi)$. Suppose also that $p$ is not contained in the closure of the image of any rational section of $\pi$ (notice that if $q$ is a smooth point of $B$, then every rational section passing through $p$ is actually regular at $q=\pi(p)$ since there are no $\pi$-contracted rational curves meeting $p)$. Then a very general triangle passing through $q=\pi(p)$ admits no stable sections passing through $p$.

Proof.

It suffices to exhibit a single triangle with this property. We will show directly that this holds for a very general triangle with a vertex at $q=\pi(p)$. Choose a subfamily $H \subset L$ of curves passing through $q$, such that for general $b \in B$ a finite (but positive) number of members of $H$ pass through $b$. We construct a subset $\Omega \subset X$ which is a countable union of subvarieties of $X$ in the following way. For every finite type family of sections of $\pi$ over curves in $H$ and which take the value $p$ at $q$, we have a map from the base of this family to $\bar{M}(X)$. Form the closure of the image of this map, and define $\tilde{\Omega}$ to be the countable union of all such closed subvarieties of $\bar{M}(X)$ arising from the countably many Chow varieties of sections as above. Notice that $\tilde{\Omega}$ is not necessarily quasi-compact, but it is a closed subvariety of $\bar{M}(X)$ (which is also not quasi-compact).

We can restrict the universal curve of $\bar{M}(X)$ over $\tilde{\Omega}$, and there is a map from the total space of this universal curve to $X$. We define $\Omega$ to be the image of this map, so $\Omega$ is a countable union of closed subvarieties of $X$. Let $\tilde{\Omega}_{0}$ be any irreducible component of $\tilde{\Omega}$ and let $\Omega_{0} \subset X$ be the (closed) image of $\tilde{\Omega}_{0}$.

Consider the restricted morphism $\bar{M}(\pi): \tilde{\Omega}_{0} \rightarrow \bar{M}(B)$. The general point of $\Omega_{0}$ parametrizes a section over a member of $H$, so the image of $\Omega_{0}$ under $\bar{M}(\pi)$ is contained in the closure $\bar{H}$ of $H$. And by Lemma 3.2, the morphism $\bar{M}(\pi)$ : $\Omega_{0} \rightarrow \bar{H}$ is generically finite. Therefore the map from the universal curve over $\Omega_{0}$ to the universal curve over $\bar{H}$ is generically finite. By construction, the evaluation morphism from the universal curve over $\bar{H}$ to $B$ is generically finite. So finally we conclude the restricted morphism $\pi: \Omega_{0} \rightarrow B$ is generically finite, i.e. for a general 
point $b \in B$ there are only finitely many preimages of $b$ in $\Omega_{0}$. Moreover, each of these finitely many preimages lies on an honest section over a curve in $H$ passing through $b$. So in fact $\pi: \Omega_{0} \rightarrow B$ is unramified over $b$. Since $\Omega$ is the union of countably many sets $\Omega_{0}$, we conclude that for a very general point $b \in B$ there are only countably many preimages of $b$ in $\Omega$. Moreover, if we choose $b$ very general, then every irreducible component $\Omega_{0}$ whose image $\pi\left(\Omega_{0}\right)$ contains $b$ actually surjects to $B$. Since also $\pi: \Omega_{0} \rightarrow B$ is unramified over $b$, we conclude that $\pi: \Omega_{0} \rightarrow B$ is étale over $b$. Thus for $b \in B$ a very general point, for every irreducible component $\tilde{\Omega}_{0}$ of $\tilde{\Omega}$, the restricted map $\pi: \Omega_{0} \rightarrow B$ is étale over $b$ - possibly for the trivial reason that $b$ is not contained in $\pi\left(\Omega_{0}\right)$.

Next we observe that any rational section $\rho: B \rightarrow \Omega$ includes $p$ (i.e. the closure of the image of $\rho$ contains $B$ ). First observe that $\rho$ factors through one of the subsets $\Omega_{0} \subset \Omega$. Now for a general point $b$ in $B, \pi: \Omega_{0} \rightarrow B$ is unramified over $b$. Therefore $\rho: B \rightarrow \Omega_{0}$ is actually regular in a neighborhood of $b$. And the image $\rho(b)$ lies on some honest section $\tilde{h}: C \rightarrow X$ over a curve $C$ in $H$ which contains $q$ and $b$ and such that $\tilde{h}(q)=p$. Since $\pi: \Omega_{0} \rightarrow B$ is unramified at $\rho(b)=\tilde{h}(b)$, we have that $\tilde{h}: C \rightarrow X$ and $\left.\rho\right|_{C}: C \rightarrow X$ are equal as rational maps. By the valuative criterion of properness, we conclude that $\tilde{h}: C \rightarrow X$ factors through the closure of the image of $\rho$, in particular $p=\tilde{h}(q)$ lies on the closure of the image of $\rho$. One of our hypotheses is that $p$ does not lie on the closure of the image of any rational section. Therefore we conclude that there is no rational section of $\pi$ whose image is contained in $\Omega$.

By the last paragraph, for each irreducible piece $\Omega_{0}$ of $\Omega$, we have that $\Omega_{0} \rightarrow B$ admits no rational section. Moreover, over a very general point $b \in B$, every one of the maps $\pi: \Omega_{0} \rightarrow B$ is étale. So by the proof of Lemma 3.1, for a very general curve $C_{3}$ in $L$ passing through a very general point $b \in B$ (and not necessarily passing through $q$ ), for each irreducible piece $\Omega_{0}$ of $\Omega$, there is no section of $\pi$ : $\pi^{-1}\left(C_{3}\right) \rightarrow C_{3}$ whose image lies in $\Omega_{0}$. So there is no section of $\pi: \pi^{-1}\left(C_{3}\right) \rightarrow C_{3}$ whose image lies in $\Omega$.

Since $p$ is not in $V(\pi)$, no section through $p$ can be contained in $V(\pi)$. It follows that for a very general point $b$ in $B, \Omega \cap V(\pi) \cap \pi^{-1}(b)=\emptyset$. Choose a very general curve $C_{3}$ in $L$ as above, and choose two very general points $r$ and $s$ on $C_{3}$. Then $\Omega \cap \pi^{-1}(r)$ is a countable set, disjoint from $V(\pi)$, and every point in this set lies on an honest section over a curve in $H$ passing through $r$. Given any point in this set, there are at most countably many sections of $\pi$ over $C_{3}$ which take this value at $r$. Hence, there is a countable collection of sections of $\pi$ over $C_{3}$ whose value at $r$ is contained in $\Omega$. Any such section is not contained in $\Omega$, and thus meets $\Omega$ in at most countably many points with countably many images in $C_{3}$. Choosing $s$ not to lie in any of these countably many countable sets, we conclude that for any section $\tilde{h}_{3}$ of $\pi$ over $C_{3}$ such that $\tilde{h}_{3}(r)$ is contained in $\Omega$, we have that $\tilde{h}_{3}(s)$ is not contained in $\Omega$.

Now we take our triangle to be $C=C_{1} \cup C_{2} \cup C_{3}$ where $C_{1}$ and $C_{2}$ are members of $H$ which join $q$ to $r$ and $q$ to $s$ respectively. By way of contradiction, suppose there is a stable section $\tilde{h}$ of $\pi$ over $C$ whose image contains $p$. As we have discussed, 
such a stable section consists of honest sections $\tilde{h}_{1}, \tilde{h}_{2}$ and $\tilde{h}_{3}$ over $C_{1}, C_{2}$, and $C_{3}$ respectively, along with some trees of $\pi$-contracted rational curves attached which connect $\tilde{h}_{1}(r)$ to $\widetilde{h}_{3}(r)$, which connect $\tilde{h}_{2}(s)$ to $\tilde{h}_{3}(s)$ and which connect $\tilde{h}_{1}(q)$ and $\tilde{h}_{2}(q)$ to $p$, if these points don't already coincide. By the definition of $\Omega$, the images $\tilde{h}_{1}\left(C_{1}\right)$ and $\tilde{h}_{2}\left(C_{2}\right)$ are necessarily contained in $\Omega$. Since $r$ and $s$ were chosen to be very general, there are no $\pi$-contracted rational curves over $r$ or $s$ which meet $\Omega$, in particular, there is no tree of $\pi$-rational curves which meets either $\tilde{h}_{1}(r)$ or $\tilde{h}_{2}(s)$. So we must have $\tilde{h}_{1}(r)=\tilde{h}_{3}(r)$ and $\tilde{h}_{2}(s)=\tilde{h}_{3}(s)$. Since $p$ is not contained in $V(\pi)$, also there is no tree of $\pi$-contracted rational curves which meets $p$. Therefore $\tilde{h}_{1}(q)=\tilde{h}_{2}(q)=p$. The upshot is that, after pruning any extraneous trees of $\pi$-contracted rational curves, we have that $\tilde{h}$ is an honest section of $\pi$ over the reducible curve $C$.

But now we have our contradiction: we have seen that for any section $\tilde{h}_{3}$ of $\pi$ over $C_{3}$ such that $\tilde{h}_{3}(r)$ is contained in $\Omega$, then $\tilde{h}_{3}(s)$ is not contained in $\Omega$. On the other hand we have by the last paragraph that $\tilde{h}_{3}(r)=\tilde{h}_{1}(r)$ is contained in $\Omega$ and also $\tilde{h}_{3}(s)=\tilde{h}_{2}(s)$ is contained in $\Omega$. Therefore we conclude there is no stable section $\tilde{h}$ of $\pi$ over $C$.

Of course this lemma doesn't tell us much in case the fibers of $\pi$ are uniruled. Thanks to a construction of Kollár-Miyaoka-Mori and using Theorem 1.1, we can always reduce to the case that the fibers of $\pi$ are non-uniruled.

Definition 3.7. Given a morphism $\pi: X \rightarrow B$, the relative mrc fibration

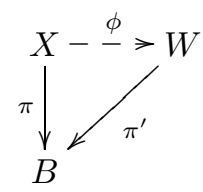

is a dominant rational map $\phi: X \rightarrow W$ of varieties over $B$ such that a general fiber of $\phi$ is rationally connected and a general fiber of $\pi^{\prime}$ is not uniruled.

The existence of the relative mrc fibration is established in [K, theorem IV.5.9], although the equivalence of the definition given there with the one above requires Theorem 1.1 .

Before applying Lemma 3.6 to our main theorem, we note a corollary which is interesting in its own right.

Corollary 3.8. If $B$ is a normal, quasi-projective variety, and $\mathcal{H}$ is any family of curves in $B$ which dominates $T(L)$ and whose general member is smooth, then for any projective morphism $\pi: X \rightarrow B$, the following two conditions are equivalent:

(1) A general point of $X$ lies in a pseudosection.

(2) For a general curve $C$ parametrized by $\mathcal{H}$, a general point of $X_{C}=\pi^{-1}(C)$ lies on a section of $\pi: X_{C} \rightarrow C$.

Note that the existence of such a family $\mathcal{H}$ is ensured by Lemma 2.S. 
Proof.

One direction follows from a stronger version of Theorem 1.1 which states that if $\pi: Z \rightarrow C$ has rationally connected general fiber there is a section of $\pi$ through a general point of $Z$ (cf. [K, IV.6.10]). The other direction is more interesting. If the general fiber of $\pi$ is not uniruled then this follows from Lemma 3.6, since in this case $X$ being generically covered by pseudosections is equivalent to it being generically covered by rational sections. Therefore, suppose that the general fiber of $\pi$ is uniruled. Consider the relative mrc fibration of $\pi: X \rightarrow B$,

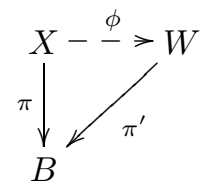

The two conditions we are trying to prove equivalent are both preserved under blowing up of $X$ (to see this for the second condition, restrict to the general case that $C$ is smooth). Thus we are free to blow up $X$ in order to resolve the indeterminacy locus of $\phi$. So we may assume that $\phi$ is actually a regular morphism. Suppose that there is a section through a general point of $X_{C}$ over a general curve $C$. Composing with $\phi$ gives us a section of $W_{C}$ through a general point of $W_{C}$ over a general curve $C$. Since the fibers of $\pi^{\prime}$ are not uniruled, we conclude by the last paragraph that a general point of $W$ is contained in a rational section of $\pi^{\prime}$. Taking the preimage of this rational section under $\phi$ gives us a pseudosection of $\pi$ and passing through a general point.

Our proof of Theorem 1.3 proceeds similarly. First we will prove the result for maps whose general fiber is not uniruled, and then we will handle the general case by appealing to the relative mrc fibration. In addition we will use an induction on the relative dimension of $X$ over $B$. We have already considered the case of fiber dimension zero in Proposition 3.1, thus suppose that $d>0$. By way of induction, assume that we have already constructed a family $\mathcal{H}_{d-1}$ of smooth curves in $B$ which cover $B$ and such that for any morphism $\pi: X \rightarrow B$ of relative dimension less than $d, \pi$ admits a section when restricted over a very general curve in $\mathcal{H}_{d-1}$ if and only if $\pi$ admits a pseudosection. We construct $\mathcal{H}_{d}$ as follows. First we construct a family of reducible nodal curves by letting $\tilde{\mathcal{H}}_{d}$ be the family of maps $f: C \rightarrow X$ of the form $C=C_{0} \cup C_{1} \cup \cdots \cup C_{d+1}$, where $\left[f_{C_{0}}\right]$ is a member of $\mathcal{H}_{d-1}$ and the other $C_{i}, i=1, \ldots, d+1$ are triangles which are pairwise disjoint and which each meet $C_{0}$ in a single node which is embedded in the smooth locus of $B$. Now take $\mathcal{H}_{d}$ to be any family of smooth curves which dominates $\tilde{\mathcal{H}}_{d}$. By Lemma 2.3 . we can take $\mathcal{H}_{d}$ to be the family of linear sections of $B$ under a sufficiently positive projective embedding.

We need to check that $\mathcal{H}_{d}$ satisfies the desired property. Namely, suppose $\pi$ : $X \rightarrow B$ is a projective morphism of relative dimension less than or equal to $d$ which does not admit a pseudosection. Then we need to show that over a very general member of $\mathcal{H}_{d}, \pi$ does not admit a section. By Lemma 2.5, it suffices to check that over a very general member of $\tilde{\mathcal{H}}_{d}, \pi$ does not admit a stable section. 
First we will consider the case where the general fiber of $\pi$ is not uniruled. In order to later handle the uniruled case, it will be useful for us to prove a statement that seems stronger than necessary. As above, we let $V(\pi)$ be the rational curve locus which is the union of all $\pi$-contracted rational curves in $X$, which is a subset of $X$ which is a countable union of subvarieties. We let $Y(\pi)$ be the union of $V(\pi)$ and all rational sections of $\pi$. This is also a countable union of subvarieties of $X$. Note that Lemma 3.6 says exactly that for any point $p$ in $X \backslash Y(\pi)$, a very general triangle through $\pi(p)$ admits no stable sections containing $p$.

Lemma 3.9. Any stable section of $\pi$ over a very general member of $\tilde{\mathcal{H}}_{d}$ has values over $C_{0}$ contained in $Y(\pi)$.

Before proving the lemma, we remark that (given the inductive hypothesis) it immediately implies our theorem in the case where the fibers are not uniruled. If $\pi: X \rightarrow B$ is a morphism whose general fiber is not uniruled and which does not admit a pseudosection then $Y(\pi)=V(\pi)$ is a countable union of proper subvarieties of $X$, i.e. it is a countable union of subvarieties $Y(\pi)_{0}$ of $X$ such that the fiber dimension of $Y(\pi)_{0} \rightarrow B$ is strictly less than $d$. By the induction assumption, for a very general $C_{0}$ in $\mathcal{H}_{d-1}$, there can be no honest section of $\pi$ contained in any of the subvarieties $Y(\pi)_{0}$. Thus, by the lemma, there can be no stable section of $\pi$ over $C=C_{0} \cup C_{1} \cup \cdots \cup C_{d+1}$.

Proof.

We now prove the lemma. We imagine assembling our very general member of $\tilde{\mathcal{H}}_{d}$ one component at a time. Pick a very general $C_{0} \in \mathcal{H}_{d-1}$ and let $\Sigma$ denote the parameter space of all sections of $\pi$ over $C_{0}$ which are not contained in $Y(\pi)$. This is the complement of a countable union of subvarieties in a countable union of subvarieties of $\bar{M}(X)$. Denote the irreducible components of $\Sigma$ by $\Sigma^{\alpha}$, and by Lemma 3.4 we conclude that each $\Sigma^{\alpha}$ has dimension less than or equal to $d$.

Our strategy now is simple. The condition that a section over $C_{0}$ extends to a section over $C_{0} \cup C_{i}$ should impose a condition by Lemma 3.6, and so after imposing $d+1$ conditions there should be no sections left. To prove this, we consider the chain

$$
\Sigma_{d+1} \subset \Sigma_{d} \subset \cdots \subset \Sigma
$$

where $\Sigma_{i}$ is defined to be the subset of $\Sigma$ parametrizing sections of $\pi$ over $C_{0}$ which are not contained in $Y(\pi)$ and which can extend over $C_{0} \cup C_{1} \cup \cdots \cup C_{i}$. That is, if we let $q_{1}, \ldots, q_{d+1}$ be the very general points at which we attach the triangles, $\Sigma_{i}$ parametrizes those sections of $\pi$ over $C_{0}$ whose value at $q_{j}$ agrees with the value of some stable section of $\pi$ over $C_{j}$ for all $j \leq i$. We will prove by induction on $i$ that $\operatorname{dim}\left(\Sigma_{i}\right) \leq d-i$ for each $i$, in particular $\Sigma_{d+1}$ is empty.

We have already seen that every component of $\Sigma=\Sigma_{0}$ has dimension at most $d$, so this establishes the base case $i=0$. By way of induction, assume that every component of $\Sigma_{k}$ has dimension at most $d-k$. Now we want to show the result for $k+1$. For any one of the countably many irreducible components $\Sigma_{k}^{\alpha}$ of $\Sigma_{k}$, for a general point $q_{k+1}$ some section $\tilde{h}_{0}^{\alpha}$ parametrized by $\Sigma_{k}^{\alpha}$ maps $q_{k+1}$ to a point not 
contained in $Y(\pi)$, i.e. the point $p^{\alpha}=\tilde{h}_{0}\left(q_{k+1}\right)$ is not in $Y(\pi)$. So if we choose a very general point $q_{k+1}$ we can arrange that for every irreducible component $\Sigma_{k}^{\alpha}$ of $\Sigma_{k}$, there is a section $\tilde{h}_{0}^{\alpha}$ in $\Sigma_{k}^{\alpha}$ such that $p^{\alpha}=\tilde{h}_{0}^{\alpha}\left(q_{k+1}\right)$ is not contained in $Y(\pi)$.

Now for each $\alpha$, for a general triangle $C_{k+1}$ through $q_{k+1}$, we conclude by Lemma 3.6 that there is no stable section over $C_{k+1}$ which passes through $p^{\alpha}$. So if we choose a very general triangle $C_{k+1}$, we can arrange that for every $\alpha$, there is no stable section over $C_{k+1}$ which passes through any of the points $p^{\alpha}$. So none of the sections $\tilde{h}_{0}^{\alpha}$ extends to a stable section over $C \cup C_{k+1}$. So for each $\alpha$, $\Sigma_{k+1} \cap \Sigma_{k}^{\alpha}$ is a proper closed subvariety and thus has dimension strictly less than $\operatorname{dim}\left(\Sigma_{k}^{\alpha}\right) \leq d-k$. Since we have

$$
\Sigma_{k+1}=\cup_{\alpha}\left(\Sigma_{k+1} \cap \Sigma_{k}^{\alpha}\right)
$$

we conclude that every irreducible component of $\Sigma_{k+1}$ has dimension at most $d-$ $k-1$. So the claim is proved by induction on $k$. In particular, we conclude that $\Sigma_{d+1}=\emptyset$, i.e. over $C_{0}$ every section of $\pi$ which can be extended to stable sections over $C$ is contained in $Y(\pi)$.

As discussed above, Lemma 3.9 proves the induction step in case the fibers of $\pi$ are not uniruled. So to finish the inductive proof of Theorem 1.3, we are left to consider the case where the fibers of $\pi$ are uniruled. We argue by contradiction.

By way of contradiction, assume that we have a morphism $\pi: X \rightarrow B$ with no pseudosection, but which admits a section when restricted to every element of $\mathcal{H}_{d}$. Let $\phi: X \rightarrow W$ be the relative mrc fibration. We may resolve the indeterminacy locus of $\phi$ by blowing up:

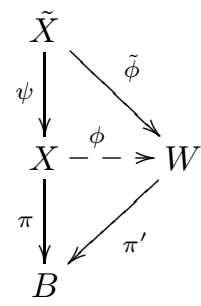

Let $E \subset X$ denote the fundamental locus of the morphism $\psi$ (i.e. the image under $\psi$ of the exceptional divisor of $\psi$ ).

Notice that the relative dimension of $\left.\pi\right|_{E}: E \rightarrow B$ is strictly less than $d$. Suppose that $\left.\pi\right|_{E}: E \rightarrow B$ admits a section when restricted over a very general curve $C$ in $\mathcal{H}_{d}$. By Lemma 2.5, we conclude that $\left.\pi\right|_{E}: E \rightarrow B$ admits a stable section when restricted over a stable map in $\tilde{\mathcal{H}}_{d}$. In particular, since every curve in $\mathcal{H}_{d-1}$ occurs as the $C_{0}$-submap of a stable map in $\tilde{\mathcal{H}}_{d}$, we conclude that $E \rightarrow B$ admits a section when restricted over a very general curve $C_{0}$ in $\mathcal{H}_{d-1}$. By the induction hypothesis, this implies that there is a pseudosection of $\left.\pi\right|_{E}: E \rightarrow B$. But, in particular, this implies there is a pseudosection of $\pi: X \rightarrow B$ which contradicts our assumption. So we conclude that for a very general curve $C$ in $\mathcal{H}_{d}, \pi_{E}: E \rightarrow B$ admits no section when restricted over $C$. On the other hand, our assumption is 
that $\pi: X \rightarrow B$ does admit a section over $C$, i.e. there exists a section over $C$ which is not contained in $E$. This is the same as a rational section of $\pi \circ \psi: \tilde{X} \rightarrow B$ over $C$. Since $C$ is smooth, by the valuative criterion of properness this rational section of $\pi \circ \psi$ extends to a regular section of $\pi \circ \psi$ over $C$.

Thus we find that $\pi \circ \psi$ admits a section over a very general curve $C$ in $\mathcal{H}_{d}$. Now if $\pi \circ \psi$ admits a pseudosection, so does $\pi$ by simply taking the image of the pseudosection under $\psi$. Therefore we conclude that $\pi \circ \psi: \tilde{X} \rightarrow B$ admits no pseudosection, but it does admit a section when restricted over a very general curve in $\mathcal{H}_{d}$. Therefore, as far as deriving a contradiction is concerned, we can replace $X$ by $\tilde{X}$. So from now on we assume that $\phi: X \rightarrow W$ is a regular morphism.

Let $W^{\prime} \subset W$ denote the locus of points over which the fiber of $\phi$ is not rationally connected. Any rational section of $\pi^{\prime}$ not contained in $W^{\prime}$ gives rise to a pseudosection of $\pi$, which doesn't exist by hypothesis. Therefore all rational sections of $\pi^{\prime}$ are contained in $W^{\prime}$. Applying Lemma 3.9, we find that over a general member $C$ of $\tilde{\mathcal{H}}_{d}$, any stable section of $\pi^{\prime}$ maps $C_{0}$ into the subset $W^{\prime} \cup V\left(\pi^{\prime}\right)$. Thus, any stable section of $\pi$ over $C$ has maps $C_{0}$ into the subset $\phi^{-1}\left(W^{\prime} \cup V\left(\pi^{\prime}\right)\right)$.

On the other hand, $\phi^{-1}\left(W^{\prime} \cup V\left(\pi^{\prime}\right)\right)$ is a countable union of proper subvarieties of $X$, each of which has relative dimension at most $d-1$ over $B$. So by the induction hypothesis, every section of $\pi: X \rightarrow B$ over a very general curve in $\mathcal{H}_{d-1}$ has image which is not contained in $\phi^{-1}\left(W^{\prime} \cup V\left(\pi^{\prime}\right)\right)$. Of course $C_{0}$ is a very general curve in $\mathcal{H}_{d-1}$, and so admits no sections in this locus. So we conclude that over a

very general member of $\tilde{\mathcal{H}}_{d}, \pi$ admits no stable section. By Lemma 2.5, $\pi$ admits no section over a very general member of $\mathcal{H}_{d}$, and this is a contradiction of our assumptions.

So, in the case that the fibers of $\pi$ are uniruled, we have established the induction step by contradiction. We had previously established the induction step in the case of non uniruled fibers. This finishes the proof of the induction step, so the main theorem is proved by induction.

\section{THe General CASE}

In the last section we proved the main theorem in case $B$ is normal and quasiprojective and $\pi: X \rightarrow B$ is projective. In this section we will show how to reduce the general case to this case. We proceed by induction on the dimension.

Suppose that $B$ is a finite type algebraic variety. Then by Chow's lemma we can find a projective, birational morphism $B_{1} \rightarrow B$ such that $B_{1}$ is quasi-projective. Now by Noether normalization, the normalization $B_{2} \rightarrow B_{1}$ of $B_{1}$ is a finite morphism. Thus $f: B_{2} \rightarrow B$ is a projective, birational morphism such that $B_{2}$ is quasi-projective and normal. Let $\mathcal{H}_{d}$ be the family of curves $C$ in $B_{2}$ constructed in the last section. The restriction of $f$ to a general curve in this family - let's call this restriction $h: C \rightarrow B_{2}-$ is a nonconstant morphism, i.e. it is a stable 
map. Therefore, replacing $\mathcal{H}_{d}$ by a Zariski dense open subset, we may consider $\mathcal{H}_{d}$ to be a family of stable maps $h: C \rightarrow B$ with smooth domain. The claim is that Theorem 1.3 holds for $B$ and $\mathcal{H}_{d}$. We will prove this by induction, but before proceeding to the induction argument we introduce a little more notation.

Suppose that $\pi: X \rightarrow B$ is a proper morphism of relative dimension at most $d$ which admits no pseudosection. We need to prove that for a very general map $h: C \rightarrow B$ in $\mathcal{H}_{d}, \pi$ admits no section over $h$. The base change $\pi_{2}: X \times_{B}$ $B_{2} \rightarrow B_{2}$ is a proper morphism of relative dimension at most $d$ which admits no pseudosection, since the image under $\pi_{1}: X \times_{B} B_{2} \rightarrow X$ of a pseudosection of $\pi_{2}$ is a pseudosection of $\pi$. By again applying Chow's lemma, we can find a projective, birational morphism $\phi: X_{2} \rightarrow X$ such that $\pi_{2} \circ \phi: X_{2} \rightarrow B_{2}$ is projective. Any pseudosection of $\pi_{2} \circ \phi$ maps under $\phi$ to a pseudosection of $\pi_{2}$. Therefore $\pi_{2} \circ \phi$ admits no pseudosection.

Now $\pi_{2} \circ \phi: X_{2} \rightarrow B_{2}$ satisfies the hypotheses of the last section. By the proof of the main theorem in that section, for a very general curve $C$ in $\mathcal{H}_{d}, \pi_{2} \circ \phi$ admits no section over $C$. Let $Z \subset X$ denote the fundamental locus of the birational, projective morphism $X_{2} \rightarrow X$, i.e. the locus over which this morphism is not an isomorphism.

If $d=0$, we are essentially done. The locus $Z \subset X$ is a proper subvariety, and since $\pi$ is generically finite, also $\pi(Z) \subset B$ is a proper subvariety. If we choose a very general map $h: C \rightarrow B$ in $\mathcal{H}_{0}$, then the image $h(C)$ does not lie in $\pi(Z)$. But then any section of $\pi: X \rightarrow B$ over $h$ determines a rational section of $\pi_{2} \circ \phi: X_{2} \rightarrow B_{2}$ over $C$. Since $C$ is smooth, by the valuative criterion of properness this rational section extends to a regular section. This contradicts the result of the last section. So we conclude that for a very general map $h: C \rightarrow B$ in $\mathcal{H}_{0}$, there is no section of $\pi: X \rightarrow B$ over this map.

Now we proceed by induction. We have established the base case $d=0$, so we suppose that $d>1$. By way of induction, we suppose the theorem has been proved for $d-1$. Consider $\left.\pi\right|_{Z}: Z \rightarrow B$. This morphism has fiber dimension at most $d-1$. By our induction assumption, we conclude that $\left.\pi\right|_{Z}: Z \rightarrow B$ has no section when restricted over a very general map $h_{0}: C_{0} \rightarrow B$ in $\mathcal{H}_{d-1}$. By Lemma 2.5, we conclude that $\left.\pi\right|_{Z}: Z \rightarrow B$ has no section when restricted over a very general map $h: C \rightarrow B$ in $\mathcal{H}_{d}$ (since $\mathcal{H}_{d}$ dominates $\mathcal{H}_{d-1}$ ). So if we choose a very general map $h: C \rightarrow B$ in $\mathcal{H}_{d}$, then for any section $\tilde{h}: C \rightarrow X$ of $\pi$ over $h$, we have that $\tilde{h}(C)$ is not contained in $Z$. So the regular section $\tilde{h}$ determines a rational section of $\pi_{2}: X_{2} \rightarrow B_{2}$ over $C$. Since $C$ is smooth, by the valuative criterion of properness this rational section extends to a regular section. This contradicts the result of the last section. So we conclude that for a very general map $h: C \rightarrow B$ in $\mathcal{H}_{d}$, there is no section of $\pi: X \rightarrow B$ over $h$.

\section{Application: families of Enriques surfaces}

In this section we'll show how to apply Theorem 1.3 to a family of Enriques surfaces to deduce Corollary 1.4, that is, to find a one-parameter family of Enriques surfaces without a section. 
5.1. A family of quartic Enriques surfaces. The family we'll be starting with is the universal family over a parameter space for quartic Enriques surfaces: that is, a family of polarized Enriques surfaces $S$ with a polarization $M \in \operatorname{Pic}(S)$ of self-intersection 4 that includes a general such surface. Now, for the purposes of applying Theorem 1.3 and deducing Corollary 1.4, we can just write down the family as in Definition 5.2 below - we don't need to know that it is actually the generic quartic Enriques surface, and the reader who doesn't particularly care can jump directly to Definition 5.2 - but since we're going to be working closely with the family it seems worthwhile to take a few paragraphs and establish its bonafides.

To begin with, since Enriques surfaces $S$ have fundamental group $\pi_{1}(S) \cong \mathbb{Z} / 2 \mathbb{Z}$ and have as universal covering space a K3 surface, a quartic Enriques surface $S$ is the quotient of an octic K3 surface $T$ - that is, a K3 surface $T$ with a polarization $L \in \operatorname{Pic}(T)$ of self-intersection $c_{1}(L)^{2}=8$ - by an involution $\tau$ of $T$ preserving $L$. For a generic octic K3 $(T, L)$, the linear system of sections of $L$ is base-point-free and defines an embedding of $T$ into $\mathbb{P}^{5}$, and the image surface is the intersection of three quadric hypersurfaces in $\mathbb{P}^{5}$ with defining equations $Q_{1}, Q_{2}$, and $Q_{3}$.

Next, since $\tau^{*} L \cong L$, the action of $\tau$ can be lifted to an action on $H^{0}(T, L)$, and hence to an involution of $\mathbb{P}^{5}$ carrying $T$ to itself. Moreover, since by Riemann-Roch

$$
h^{0}(S, M)=\frac{c_{1}(M)^{2}}{2}+\chi\left(\mathcal{O}_{S}\right)=\frac{4}{2}+1=3
$$

the action of $\tau$ on $H^{0}(T, L)$ must have eigenvalues 1 and -1 , each with multiplicity 3 . We thus have a canonical direct-sum decomposition

$$
H^{0}(T, L)=\Gamma \oplus \Psi
$$

with $\operatorname{dim} \Gamma=\operatorname{dim} \Psi=3$.

Applying the same principle, we see that the action of $\tau$ on $H^{0}\left(T, L^{2}\right)$ has eigenvalue 1 with multiplicity

$$
h^{0}\left(S, M^{2}\right)=\frac{c_{1}\left(M^{2}\right)^{2}}{2}+\chi\left(\mathcal{O}_{S}\right)=\frac{16}{2}+1=9
$$

and correspondingly eigenvalue -1 with multiplicity $h^{0}\left(T, L^{2}\right)-9=18-9=9$. On the other hand, given that $H^{0}(T, L)=\Gamma \oplus \Psi$ as above, we can write

$$
\operatorname{Sym}^{2} H^{0}(T, L)=\operatorname{Sym}^{2} \Gamma \oplus(\Gamma \otimes \Psi) \oplus \operatorname{Sym}^{2} \Psi
$$

with the action of $\tau$ on $\operatorname{Sym}^{2} H^{0}(T, L)$ having (+1)-eigenspace $\operatorname{Sym}^{2} \Gamma \oplus \operatorname{Sym}^{2} \Psi$ of dimension 12 and $(-1)$-eigenspace $\Gamma \otimes \Psi$ of dimension 9. It follows that the kernel of the restriction map

$$
\operatorname{Sym}^{2} H^{0}(T, L) \longrightarrow H^{0}\left(T, L^{2}\right)
$$

- that is, the vector space of quadrics in $\mathbb{P}^{5}$ vanishing on $T \subset \mathbb{P}^{5}$ - must be contained in the direct sum $\operatorname{Sym}^{2} \Gamma \oplus \operatorname{Sym}^{2} \Psi$. In other words, we can choose homogeneous coordinates

$$
[Z, W]=\left[Z_{0}, Z_{1}, Z_{2}, W_{0}, W_{1}, W_{2}\right]
$$

on $\mathbb{P}^{5}$ so that the action of $\tau$ is given by

$$
\tau:\left[Z_{0}, Z_{1}, Z_{2}, W_{0}, W_{1}, W_{2}\right] \mapsto\left[Z_{0}, Z_{1}, Z_{2},-W_{0},-W_{1},-W_{2}\right]
$$


and the defining equations of the double cover $T$ of a general quartic Enriques surface $S$ may be written in the form

$$
Q_{\alpha}(Z, W)=Q_{\alpha}^{\prime}(Z)+Q_{\alpha}^{\prime \prime}(W) .
$$

We are now prepared to write down the families of K3 and Enriques surfaces we'll be studying in the sequel. To start with, let $\Gamma$ and $\Psi$ be 3 -dimensional vector spaces, denote by $\mathbb{P}^{5}$ the projective space of 1-dimensional subspaces of $\Gamma \oplus \Psi$ And let

$$
\mathbb{P}^{11}=\mathbb{P}\left(\operatorname{Sym}^{2} \Gamma^{\vee} \oplus \operatorname{Sym}^{2} \Psi^{\vee}\right)
$$

be the projective space of 1-dimensional subspaces of the (12-dimensional) vector space of quadrics on $\mathbb{P}^{5}$ of the form above. Finally, we let $[Z, W]=\left[Z_{0}, Z_{1}, Z_{2}, W_{0}, W_{1}, W_{2}\right]$ be homogeneous coordinates on $\mathbb{P}^{5}$ with $\Gamma$ the zero locus of $W_{0}, W_{1}$ and $W_{2}$, and $\Psi$ likewise the zero locus of $Z_{0}, Z_{1}$ and $Z_{2}$; and we let $\tau$ be the involution $\left[Z_{0}, Z_{1}, Z_{2}, W_{0}, W_{1}, W_{2}\right] \mapsto\left[Z_{0}, Z_{1}, Z_{2},-W_{0},-W_{1},-W_{2}\right]$ of $\mathbb{P}^{5}$.

Definition 5.1. By the principal family of K3 surfaces we will mean the family $\pi: Y \rightarrow B$ with $B=\mathbb{P}^{11} \times \mathbb{P}^{11} \times \mathbb{P}^{11}$ and $Y \subset B \times \mathbb{P}^{5}$ the subvariety given by

$$
Y=\left\{\left(Q_{1}, Q_{2}, Q_{3},[Z, W]\right): Q_{\alpha}(Z, W)=0 \quad \forall \alpha=1,2,3\right\},
$$

with $\pi: Y \rightarrow B$ the projection on the first factor.

Note that the action of $\tau$ on the second factor of $B \times \mathbb{P}^{5}$ carries $Y$ into itself, so that we can make the second

Definition 5.2. By the principal family of Enriques surfaces we will mean the family $\pi: X \rightarrow B$ with $B$ again as above and $X$ the quotient of the variety $Y$ above by the involution $\tau$ of $\mathbb{P}^{5}$.

It may be a misnomer to call these families of K3 and Enriques surfaces, since they are only generically that: there are degenerate fibers, and even fibers of dimension greater than 2. But it's convenient to use the term, and we hope the reader will forgive this.

5.2. Proof of Corollary 1.4. In order to apply Theorem 1.3 to the principal family of Enriques surfaces and deduce Corollary 1.4, we simply have to show that $X \rightarrow B$ admits no pseudosections. We'll do this by analyzing the corresponding family $Y \rightarrow B$ of K3 surfaces, since their equations are in simpler form. We start with the straightforward

Lemma 5.3. Let $Y \rightarrow B$ be the principal family of K3 surfaces of Definition 5.1. The total space $Y$ is smooth, and its Chow ring is generated by restrictions of pullbacks of hyperplane classes under the inclusion

$$
Y \hookrightarrow \mathbb{P}^{11} \times \mathbb{P}^{11} \times \mathbb{P}^{11} \times \mathbb{P}^{5} .
$$

Proof.

To start, introduce the variety

$$
W=\{(Q, p): p \in Q\} \subset \mathbb{P}^{11} \times \mathbb{P}^{5} .
$$

Via the projection $\eta: W \rightarrow \mathbb{P}^{5}$ on the second factor, $W$ is a $\mathbb{P}^{10}$-bundle over $\mathbb{P}^{5}$; it's therefore smooth, and its Chow ring is generated over the Chow ring of $\mathbb{P}^{5}$ by any class whose restriction to the fibers of $\eta$ is the hyperplane class on $\mathbb{P}^{10}$ - for 
example, the restriction of the pullback of the hyperplane class from $\mathbb{P}^{11}$, via the inclusion $W \hookrightarrow \mathbb{P}^{11} \times \mathbb{P}^{5}$. Since the total space $Y$ of our principal family of K3 surfaces is (via projection to $\mathbb{P}^{5}$ ) simply the triple fiber product

$$
Y=W \times_{\mathbb{P}^{5}} W \times_{\mathbb{P}^{5}} W
$$

the Lemma follows.

As an immediate corollary of this Lemma, we have the following description of cycles $Z \subset X$ of relative dimension 0 over $B$ :

Proposition 5.4. Let $X \rightarrow B$ be the principal family of Enriques surfaces as in Definition 5.9. If $Z \subset X$ is any cycle of codimension 2, the degree of the projection $\left.\pi\right|_{Z}: Z \rightarrow B$ is divisible by four.

Proof.

Let $\eta: Y \rightarrow X$ be the quotient map. Let $T$ be the class of a general fiber of $Y$ over $B$. By the preceding Lemma, the class of any cycle in $Y$ is a polynomial (with integer coefficients) in the restrictions to $Y$ of the pullbacks of the hyperplane classes to $\mathbb{P}^{11} \times \mathbb{P}^{11} \times \mathbb{P}^{11} \times \mathbb{P}^{5}$. But the first three of these classes restrict to 0 on a general fiber, so the class of $\eta^{-1} Z \cdot T$ must be a multiple of the restriction to $T$ of the hyperplane class on $\mathbb{P}^{5}$ This has degree divisible by eight. As $\eta$ has degree two, the Proposition follows.

As an immediate consequence of Proposition 5.4, we see that the principal family $X \rightarrow B$ of Enriques surfaces has no rational sections: the image of such a section would give a codimension 2 cycle of $X$ with degree one over $B$.

In order to show that $X \rightarrow B$ admits no pseudosections, it remains to prove that $X$ cannot contain a subvariety $Z \subset X$ whose general fiber over $B$ is an irreducible rational curve. To do this, suppose that $Z$ is such a subvariety. Let $\tilde{Z}$ be a resolution of singularities of $Z$. We then have a commutative diagram

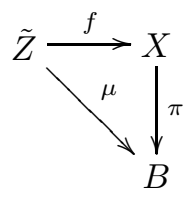

Consider the class $f_{*}\left(c_{1}\left(\omega_{\mu}\right)\right)$ in $A^{2}(X)$. Since the general fiber of $\tilde{Z}$ over $B$ is a smooth rational curve, this class has degree -2 when restricted to a general fiber of $\pi$. This contradicts the fact that all elements of $A^{2}(X)$ have degree over $B$ divisible by four.

We have thus established the

Lemma 5.5. The principal family $X \rightarrow B$ of Enriques surfaces admits no pseudosections.

Applying Theorem 1.3 we may deduce Corollary 1.4. 


\section{Application: TORSORS FOR ABELIAN VARIETIES}

It follows from Theorem 1.3 that any family $\pi: X \rightarrow B$ of smooth, connected, projective curves of positive genus over some smooth variety $B$ has a section over $B$ if and only if the restriction of this family over every curve $C \subset B$ has a section: since the fibers contain no rational curves, every pseudosection is a rational section, and every rational section is everywhere defined. Similarly, we have the following corollary:

Corollary 6.1. Let $B$ be a smooth variety, let $A \rightarrow B$ be an Abelian scheme over $B$ (i.e. a family of Abelian varieties over $B$ ), and let $\pi: T \rightarrow B$ be a torsor for $A \rightarrow B$. Then $\pi$ is a trivial torsor if and only if for every curve $C \subset B$, the restriction $T_{C} \rightarrow C$ is a trivial torsor for $A_{C} \rightarrow C$.

Since torsors for an Abelian scheme are classified by étale cohomology with coefficients in the group scheme, we can rephrase Corollary 6.1 by saying that the map

$$
H_{\text {ét }}^{1}(B, A) \rightarrow \prod_{C \subset B} H_{\text {ét }}^{1}\left(C, A_{C}\right)
$$

is injective. Note that the Lefschetz hyperplane theorem for Picard groups tells us that the same is true if we replace the Abelian scheme $A \rightarrow B$ by the commutative group scheme $\mathbb{G}_{m} \times B \rightarrow B$. It is an interesting question, for which other (possibly noncommutative) group schemes over $B$ does this hold.

\section{Further Questions AND CONJECTURES}

In this section we will consider some questions and conjectures raised by Theorem 1.3

7.1. Arithmetic question. For arithmetic questions related to rationally connected varieties, we refer the reader to GHMS. Let us just mention the following question which is the analogue of a corollary in the last section. Let $K$ be a number field, let $B$ be a smooth scheme defined over $K$, and let $\pi: X \rightarrow B$ be a proper, smooth morphism of schemes whose geometric fibers are connected curves of positive genus. Suppose that for every number field extension $L / K$, the induced mapping on rational points $\pi: X(L) \rightarrow S(L)$ is surjective - we refer to this property by saying $\pi$ is arithmetically surjective. Does it then follow that $\pi: X \rightarrow S$ has a section? We may also ask the same question when the geometric fibers of $\pi$ are Abelian varieties.

7.2. Possible extensions. We would like to take a moment here to discuss possible extensions of Theorem 1.3. To begin with, we interpreted the theorem as stating that a family of varieties $\pi: X \rightarrow B$ such that every one-parameter subfamily has a section has this property "by virtue of" the fact that $X$ contains a family of rationally connected varieties. But the statement of the theorem asserts only the existence of a pseudosection in $\pi: X \rightarrow B$; it doesn't assert any direct connection between the sections of $X_{C} \rightarrow C$ over very general curves $C$ and the pseudosection. Accordingly, we could ask:

Question 7.1. Does there exist a family $\mathcal{H}_{d}^{\prime}$ of curves on $B$, whose general member is smooth and irreducible, with the property that for any proper morphism $\pi: X \rightarrow$ 
$B$ of relative dimension $d$, for very general $[C] \in \mathcal{H}_{d}^{\prime}$ every section of the pullback

$$
\pi_{C}: X_{C}=X \times{ }_{B} C \rightarrow C
$$

lies in a pseudosection of $\pi: X \rightarrow B$ ?

One special case of this question is when $\pi: A \rightarrow B$ is an Abelian scheme over a smooth variety $B$. In this case we are asking whether we can find a family of curves $C \subset B$ such that for a very general member of this family, the map

$$
H_{\text {ét }}^{0}(B, A)-->H_{\text {êt }}^{0}\left(C, A_{C}\right)
$$

is surjective. So, in this case, the question above is an $H^{0}$-analogue of the $H^{1}$ interpretation of Theorem 1.3 .

7.3. Dependence on $d$. A simpler question is whether we can eliminate the dependence of the family $\mathcal{H}_{d}$ of curves on $d$. The answer to this seems to be "no," and while we cannot prove the impossibility it seems worthwhile to describe here the examples that lead us to this conclusion, since it may shed some light on how fast the size of the curves in $\mathcal{H}_{d}$ have to grow with $d$.

Briefly, for any $e$ we will write down families of hypersurfaces in $\mathbb{P}^{n}$ parametrized by $B=\mathbb{P}^{2}$ with the property that their restriction to any curve $C \subset \mathbb{P}^{2}$ of degree $e$ or less has a section, but which we believe to admit no pseudosections at all. To do this, let $m$ and $n$ be any integers; let $\mathbb{P}^{n}$ be projective $n$-space with homogeneous coordinates $\left[X_{0}, \ldots, X_{n}\right]$ and let $\mathbb{P}^{N}$ be the projective space parametrizing hypersurfaces of degree $m$ in $\mathbb{P}^{n}$. Let $\mathcal{X} \subset \mathbb{P}^{N} \times \mathbb{P}^{n} \rightarrow \mathbb{P}^{N}$ be the universal hypersurface of degree $m$ in $\mathbb{P}^{n}$; that is, the zero locus of the polynomial

$$
F(a, X)=\sum a_{I} X^{I}
$$

which is linear in the coordinates $a_{I}$ on $\mathbb{P}^{N}$ and of degree $m$ in the $X_{i}$. Finally, let $\mathbb{P}^{2} \hookrightarrow \mathbb{P}^{N}$ be a general map of the form

$$
\mathbb{P}^{2} \stackrel{\nu_{e}}{\longrightarrow} \mathbb{P}^{\left(\begin{array}{c}
(+2 \\
2
\end{array}\right)-1} \rightarrow \mathbb{P}^{N}
$$

where $\nu_{e}$ is a Veronese map of degree $e$ and the second map is a general linear inclusion; and let

$$
\pi: X=\mathbb{P}^{2} \times_{\mathbb{P}^{N}} \mathcal{X} \longrightarrow \mathbb{P}^{2}
$$

be the pullback of the universal hypersurface to $\mathbb{P}^{2}$ via this inclusion.

Now assume that

$$
\left(\begin{array}{c}
e+2 \\
2
\end{array}\right)=n+1
$$

and that $m$ is large. Consider the following two assertions:

- The restriction of the family $\pi: X \rightarrow \mathbb{P}^{2}$ to any curve $C \subset \mathbb{P}^{2}$ of degree $e$ or less has a section; but

- The family $\pi: X \rightarrow \mathbb{P}^{2}$ itself has no pseudosection.

The first of these assertions is straightforward to prove: under the inclusion $\mathbb{P}^{2} \hookrightarrow \mathbb{P}^{N}$, the span of a curve $C \subset \mathbb{P}^{2}$ of degree $e$ or less has dimension $\left(\begin{array}{c}e+2 \\ 2\end{array}\right)-1=n$ or less. Thus the hypersurfaces appearing as fibers of the restriction $X_{C} \rightarrow C$ of the family $\pi: X \rightarrow \mathbb{P}^{2}$ to $C$ are all linear combinations of $n$ hypersurfaces 
$G_{1}, \ldots, G_{n} \subset \mathbb{P}^{n}$, and any point of intersection of these hypersurfaces gives a section of $X_{C} \rightarrow C$.

As for the second assertion, we cannot prove it but we give a "plausibility argument" which suggests it is true. To begin with, a general fiber of $\pi: X \rightarrow \mathbb{P}^{2}$ is a general hypersurface of degree $m$ in $\mathbb{P}^{n}$; by a result of Clemens $[\mathbb{C}]$, for $m$ large this will contain no rational curves. Thus to prove the second assertion we need only show that $\pi: X \rightarrow \mathbb{P}^{2}$ has no rational sections.

Since rational sections over $\mathbb{P}^{2}$ are tricky to parametrize we will restrict to a general curve $C \subset \mathbb{P}^{2}$ of degree $e+1$, and present evidence that the restriction $X_{C} \rightarrow C$ has no section. To do this, we start by counting the dimension of the family of sections of the product $C \times \mathbb{P}^{n}$ there are of a given degree $k$ - that is, graphs of maps $C \rightarrow \mathbb{P}^{n}$ of degree $k$ - and then estimating the number of conditions it imposes on such a section to require it lies on the hypersurface $X_{C} \subset C \times \mathbb{P}^{n}$. For the first, a map $C \rightarrow \mathbb{P}^{n}$ of degree $k$ is given by a line bundle $L$ of degree $k$ on $C$, together with $n+1$ sections of $L$ up to scalars. The line bundles of degree $k$ on $C$ are parametrized by the Jacobian of $C$, which has dimension

$$
g=\left(\begin{array}{l}
e \\
2
\end{array}\right) \text {. }
$$

If $k$ is large, moreover, each such line bundle will have $k-g+1$ global sections, so the dimension of the family of maps $C \rightarrow \mathbb{P}^{n}$ of degree $k$ is

$$
g+(n+1)(k-g+1)-1=(n+1)(k+1)-n g-1 .
$$

Now let's count how many conditions it is for the graph of such a map to lie in $X_{C}$. This is straightforward: when we pull the polynomial $F(a, X)$ defining the universal hypersurface back to $C$, the coefficients pull back to section of $\mathcal{O}_{C}(e)$ and the coordinates $X_{i}$ to sections of $L$, so that the pullback of $F$ is a section of the bundle

$$
M=L^{\otimes m} \otimes \mathcal{O}(e) .
$$

The number of conditions for this section to vanish identically should thus be

$$
\begin{aligned}
h^{0}(M) & =\operatorname{deg}(M)-g+1 \\
& =k m+e(e+1)-g+1
\end{aligned}
$$

and the expected dimension of the family of sections of $X_{C} \rightarrow C$ of degree $k$ is accordingly

$$
(n+1-m) k-(n-1)(g-1)-e(e+1) .
$$

In particular, for $m$ large this is negative, suggesting that there should be no sections.

\section{REFERENCES}

[Ca] F. Campana, Connexité rationnelle des variétés de Fano, Ann. Sc. E.N.S. 25 (1992), 539-545

[C] H. Clemens, Curves on generic hypersurfaces, Ann. Sci. École Norm. Sup. (4) 19 (1986) 629-636.

[FP] W. Fulton, R. Pandharipande, Notes on stable maps and quantum cohomology, in Algebraic geometry - Santa Cruz 1995, AMS (1995), 45-96. 
[GHMS] T. Graber, J. Harris, B. Mazur, J. Starr, Arithmetic questions related to rationally connected varieties, in preparation.

[GHS] T. Graber, J. Harris, J. Starr, Families of rationally connected varieties to appear in J. Amer. Math. Soc.

[GS] A. Grothendieck and J.-P. Serre, Correspondance Grothendieck-Serre, ed. by Pierre Colmez and J.-P. Serre, Société Math. de France 2001.

[GM] M. Goresky and R. MacPherson, Stratified Morse Theory, Ergebnisse der Math. 14, Springer-Verlag, Berlin, 1988.

[K] J. Kollár, Rational Curves on Algebraic Varieties, Ergebnisse der Math. 32, SpringerVerlag, Berlin, 1996.

[KMM] J. Kollár, Y. Miyaoka, S. Mori, Rationally Connected Varieties, J. Alg. Geom. 1 (1992) 429-448.

Mathematics Department, Harvard University, 1 Oxford St., Cambridge MA 02138, USA

E-mail address: graber@math.harvard.edu

Mathematics Department, Harvard University, 1 Oxford St., Cambridge MA 02138, USA

E-mail address: harris@math.harvard.edu

Mathematics Department, Harvard University, 1 Oxford St., Cambridge MA 02138, USA

E-mail address: mazur@math.harvard.edu

Mathematics Department, M.I.T., Cambridge MA 02139, USA

E-mail address: jstarr@math.mit.edu 